\title{
Electrochemomechanical energy conversion efficiency in silica nanochannels
}

\author{
Moran Wang • Qinjun Kang
}

Received: 23 September 2009/ Accepted: 2 November 2009

(C) Springer-Verlag 2009

\begin{abstract}
The electrochemomechanical energy conversion efficiency has been investigated using a new theoretical and numerical framework for modeling the multiphysiochemical transport in long silica nanochannels. Both the chemical dissociation effects on surface charge boundary conditions and the bulk concentration enrichment caused by double layer interactions are considered in the framework. The results show that the energy conversion efficiency decreases monotonically with the increasing ionic concentration at $\mathrm{pH}=8$. For a given ionic concentration, there is an optimal channel height for the highest efficiency. The efficiency does not increase with the $\mathrm{pH}$ value monotonically, and there is an optimal $\mathrm{pH}$ value for the maximum energy conversion efficiency as the other conditions are given. The energy conversion efficiency increases with the environmental temperature. The present results may guide the design and optimization of nanofluidic devices for energy conversion.
\end{abstract}

Keywords Energy conversion - Electrokinetic transport . Nanofluidics $\cdot$ Chemical dissociation

\begin{tabular}{|c|c|}
\hline & f symbols \\
\hline$A$ & Cross-section area of channel \\
\hline$A_{\mathrm{c}}$ & $\begin{array}{l}\text { Effective area of the channel's cross section for the } \\
\text { bulk conduction current }\end{array}$ \\
\hline & Stern layer's phenomenological capacity \\
\hline & Electric conductance \\
\hline
\end{tabular}

M. Wang $(\varangle) \cdot$ Q. Kang

Computational Earth Science Group (EES-16), Earth and Environmental Sciences Division, Center for Nonlinear Study (CNLS), Theoretical Division, Los Alamos National Laboratory, Los Alamos, NM 87545, USA

e-mail: mwang@lanl.gov $c_{\mathrm{h}} \quad$ Hydrodynamic conductance

$c_{\text {str }} \quad$ Streaming conductance

D Ion diffusivity

E Electric field strength

$\mathbf{E}_{\text {ext }} \quad$ External electric field strength

$\mathbf{E}_{\text {str }}$ Streaming induced electric field strength

$e \quad$ Absolute value of proton charge

$h \quad$ Channel height

I Electrical current

$J \quad$ Ionic flux

$k \quad$ Boltzmann's constant

$n_{\mathrm{b}} \quad$ Local bulk ionic concentration

$n_{\infty} \quad$ Free streaming bulk ionic concentration

$P_{\mathrm{s}} \quad$ Wetting parameter of the channel

$p \quad$ Pressure

$\mathrm{pK} \quad$ Logarithmic dissociation constant

$Q_{\mathrm{V}} \quad$ Volume flow rate

$T$ Temperature

u Fluid velocity vector

$z \quad$ Ionic valence

\section{Greek letters}

$\alpha \quad$ Concentration enrichment coefficient

$\beta \quad$ Figure of merit

$\varepsilon_{\mathrm{r}} \varepsilon_{0} \quad$ Permittivity of the solution

$\eta \quad$ Energy conversion efficiency

$\kappa^{-1} \quad$ Debye screening length

$\lambda_{0} \quad$ Electrical conductivity of the fluid

$\lambda_{\mathrm{s}} \quad$ Specific surface conductivity

$\mu \quad$ Fluid viscosity

$\mu_{\mathrm{m}} \quad$ Ion mobility

$\rho \quad$ Fluid density

$\rho_{\mathrm{e}} \quad$ Net charge density

$\sigma \quad$ Surface charge density

$\psi \quad$ Electric potential 
$\zeta \quad$ Surface/zeta potential

$\Gamma \quad$ Surface density of chargeable sites

\section{Introduction}

When an electrolyte solution contacts with a solid surface, the surface will probably be charged through an electrochemical adsorption process (Butt et al. 2006; Squires and Quake 2005). A net charge distribution therefore forms in the solution near the charged surface, which is referred as the electrical double layer (EDL) (Li 2004; Squires and Quake 2005). As a result, a pressure-driven flow of electrolyte through a narrow channel carries net electrical charges when they accumulate at the channel ends, which actually represents an alternative means of converting mechanical energy into electrical power (Abgrall and Nguyen 2008; Pennathur et al. 2007). The notion of utilizing such an electrokinetic effect for energy conversion is not brand-new (Morrison and Osterle 1965), yet has received reawaken attention in the context of microfluidics and nanofluidics (Chein et al. 2009; Daiguji et al. 2004a, b; Davidson and Xuan 2008a; Duffin and Saykally 2008; Ren and Stein 2008; van der Heyden et al. 2006, 2007; Xie et al. 2008; Xuan and Li 2006; Yang et al. 2003). High energyconversion efficiency and high output power are the requirements for such a device to be practical. Physical modeling of electrochemomechanical energy conversion is needed to guide the design and optimizations.

The energy conversion efficiency $(\eta)$ has been modeled for microfluidic and nanofluidic devices by calculating their electrokinetic transport using the Navier-Stokes equation for the fluid flow and the Poisson-Boltzmann equation for the electrostatic charge interactions in the double layer. In the most previous studies, either a constant surface potential

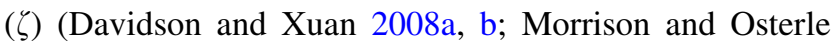
1965; Xuan and Li 2006; Yang et al. 2003) or a constant surface charge density $(\sigma)$ (Chein et al. 2009; Daiguji et al. 2004a, b; Ren and Stein 2008; van der Heyden et al. 2006) has been used as the electrical boundary condition. The constant $\zeta$ assumption led to a prediction of $\eta$ as a strong function of free streaming bulk ionic concentration $\left(n_{\infty}\right)$ in the regime of double-layer overlap (Davidson and Xuan 2008a, b; Yang et al. 2003). In contrast, $\eta$ has been found almost independent of $n_{\infty}$ when the double layer overlap occurs by assuming constant $\sigma$ on the channel surfaces (Ren and Stein 2008, van der Heyden et al. 2006). In fact, neither the constant $\zeta$ nor the constant $\sigma$ assumption could reflect the real charge status on the solid-liquid interface (Butt et al. 2006). The surface charge essentially varies with the local bulk ionic concentration $\left(n_{\mathrm{b}}\right)$, the $\mathrm{pH}$ value and the temperature $(T)$ of the solution, and the double layer interactions. The electrical boundary condition should be determined by a local chemical equilibrium on the solidliquid interface (Behrens and Grier 2001; Butt et al. 2006). van der Heyden et al. (2005) predicted the streaming conductance and the energy conversion efficiency (van der Heyden et al. 2007) of nanofluidic channels using a chemical equilibrium model and compared the results with other models and their experimental data. Choi and Kim (2009) modeled the streaming conductance of silica nanochannels using a self-consistent model and claimed that their predictions were more accurate than any models in the previous studies; however, their models still significantly deviated from the experimental data at the low concentration region. It is still a challenge to predict transport conductance that can agree well with the experimental data in both high and low ionic concentration regions (Chang and Yang 2009). To the best of the authors' knowledge, a comprehensive analysis and modeling of such an electrochemomechanical energy conversion process in nanofluidic channels has never been found up to now.

In this contribution, we are aiming to build up a theoretical and numerical framework for modeling the multiphysiochemical transport in silica nanochannels, and to study the energy conversion mechanism and its efficiency to guide the design and optimization of nanodevices. In the following sections, the mathematical models are first introduced, including the basic governing equations, the chemical equilibrium model on interfaces, the double-layer interaction effect on local ionic concentration, the electroviscous model, and the calculation formulations for the energy conversion efficiency. After validation, the mathematical framework is used to predict the electrochemomechanical energy conversion efficiencies for various cases. Advices on optimization and new designs of such energy conversion devices are therefore provided.

\section{Mathematical models}

\subsection{Governing equations}

We focus on the multiphysiochemical transport process in 2D silica long straight smooth nanochannels. The mathematical models in this work are based on the following assumptions: (i) the system is in chemical and dynamic equilibrium; (ii) the transport process is in a steady state; (iii) the channel height is much larger than the solvent molecular size; (iv) the ions in the Stern layer are rigidly attached on the surfaces and have no contribution to the ionic current; (v) the flow in the nanochannel is very slow so that the ion convection effect is negligible; (vi) the bulk ionic concentration is not too high $(<1 \mathrm{M})$ or not too low (the Debye length is smaller than ten times the channel 
width) so that the Poisson-Boltzmann (PB) model is still applicable (Wang and Chen 2008); (vii) no other chemical reactions occur at surfaces except the dissociation of silanol groups. Under these assumptions, the governing equations for the electrokinetic transport in a straight nanofluidic channel for a monovalent electrolyte solution are as follows (Li 2004, Schoch et al. 2008):

$\nabla^{2} \psi=\frac{2 e n_{\mathrm{b}}}{\varepsilon_{\mathrm{r}} \varepsilon_{0}} \sinh \left(\frac{e \psi}{k T}\right)$,

$\rho_{\mathrm{e}}=-2 e n_{\mathrm{b}} \sinh \left(\frac{e \psi}{k T}\right)$

$\nabla \cdot \mathbf{u}=0$

$\rho \mathbf{u} \cdot \nabla \mathbf{u}=-\nabla p+\mu \nabla^{2} \mathbf{u}+\rho_{\mathrm{e}} \mathbf{E}$,

where $\psi$ denotes the static electric potential, $e$ the absolute value of proton charge, $\varepsilon_{\mathrm{r}} \varepsilon_{0}$ the permittivity of the solution, $n_{\mathrm{b}}$ the bulk ionic concentration, $k$ Boltzmann's constant, $T$ the absolute temperature, $\mathbf{u}$ the fluid velocity, $\rho$ the fluid density, $p$ the pressure, $\mu$ the viscosity, $\rho_{\mathrm{e}}$ the charge density, and $\mathbf{E}$ the electric field strength. The electric field strength (E) can be either the external electric field strength $\left(\mathbf{E}_{\text {ext }}\right)$ for electroosmotic flows or the streaming induced electric field strength $\left(\mathbf{E}_{\text {str }}\right)$ for pressure-driven flows which is often referred as the cause of the electroviscosity (Levich 1962).

\subsection{Boundary conditions}

The boundary conditions are critical to modeling electrokinetic phenomena in nanofluidics. For the hydrodynamic boundary condition, we use the nonslip model at the silica surfaces. Although the slip boundaries have been adopted by some recent researches (Davidson and Xuan 2008a; Pennathur et al. 2007; Ren and Stein 2008), and have shown significant effects to improve the energy conversion efficient, a careful molecular study showed that the hydrodynamic boundary condition, slip or not, depended on the molecular interactions between fluid and solid and the channel size (Dufreche et al. 2005; Joly et al. 2004). For the dilute solution in silica nanochannels considered in this work ( $h \geqq 2 \mathrm{~nm})$, the nonslip boundary condition is still valid very well (Lorenz et al. 2008; Qiao and Aluru 2005; Wang et al. 2007a, b, c).

For the electrostatic boundary condition, in this work, we use the Basic Stern (BS) model developed by Behrens and Grier (2001) in which the silica surfaces acquire charges in contact with water by the dissociation of silanol groups (Westall and Hohl 1980):

$\mathrm{SiOH} \leftrightarrow \mathrm{SiO}^{-}+\mathrm{H}^{+}$,

so that the zeta potential $(\zeta)$ at the interface can be expressed as a function of the surface charge density $(\sigma)$ (Behrens and Grier 2001): $\zeta(\sigma)=\frac{k T}{e} \ln \frac{-\sigma}{e \Gamma+\sigma}-(\mathrm{pH}-\mathrm{p} K) \frac{k T \ln 10}{e}-\frac{\sigma}{C}$,

where $\Gamma$ is the surface density of chargeable sites, $\mathrm{p} K$ the logarithmic dissociation constant, and $C$ the Stern layer's phenomenological capacity. Equation 6 reflects the chemical nature of the silica-water interface and its charging process; however, the BS model has some limitations: (i) unsuitable for extremely acidic solutions since the protonation of doubly coordinated groups are not taken into account; (ii) unsuitable for high-salt-concentration solutions since only the surface and salt-ion interaction is not considered.

For the flat surfaces of a nanochannel, the surface charge density can be approximated using the Grahame equation, especially for the monovalent electrolyte solution (Baldessari 2008, Baldessari and Santiago 2009):

$\sigma(\zeta)=\frac{2 \varepsilon_{\mathrm{r}} \varepsilon_{0} k T \kappa}{e} \sinh \left(\frac{e \zeta}{2 k T}\right)$

where $\kappa^{-1}$ is the Debye screening length given by $\kappa=$ $\sqrt{2 z^{2} e^{2} n_{\mathrm{b}} / \varepsilon_{\mathrm{r}} \varepsilon_{0} k T}$. Solving Eqs. 6 and 7 yields the electrostatic boundary conditions at the wall surfaces of channels when there is no double layer interaction. Such a chemical equilibrium boundary condition has been employed in the previous studies (Huang and Yang 2007; van der Heyden et al. 2005). Figure 1 shows the zeta potential on an isolated flat silica surfaces contacting with the $\mathrm{NaCl}$ solution. The solid line are predicted by the BS model, and the circles are the experimental data from Gaudin and Fuerstenau (1955). The BS model predictions

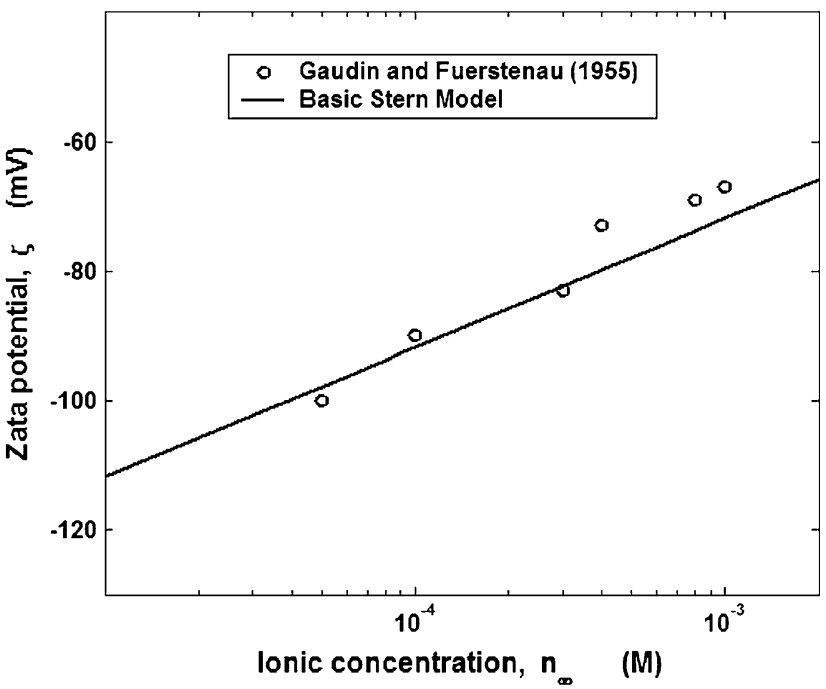

Fig. 1 Zeta potential versus bulk concentration for isolated flat silica surfaces. The cycles are from the experimental data from Gaudin and Fuerstenau (1955). The parameters using in the Basic Stern model are $\Gamma=8 \mathrm{~nm}^{-2}, C=2.9 \mathrm{~F} / \mathrm{m}^{2}$, and $\mathrm{p} K=7.5$ (Behrens and Grier 2001). The other parameters are $T=298 \mathrm{~K}$ and $\mathrm{pH}=6.5$ (Gaudin and Fuerstenau 1955) 
agree with the experimental data in the dilute solutions $\left(n_{\mathrm{b}} \leq 10^{-3} \mathrm{M}\right)$.

\subsection{Bulk concentration enrichment}

When the EDL interaction occurs in nanofluidic channels, a traditional "bulk" ionic concentration does not even exist. The local electroneutrality may be never obtained at the middle of channel for these cases. People have found the counter-ion enrichment when the EDL overlap occurs in the nanochannels (Cheng and Guo 2007, Pu et al. 2004; Wang et al. 2007a, b, c). A few methods have been proposed to determine the effective bulk ionic concentration in nanochannels (Huang and Yang 2007, Qiao and Aluru 2004). A reasonable determination for the effective bulk ionic concentration with double layer interactions in nanochannels requires (1) to reflect the dominating ions effects on transport and (2) to transform to the traditional bulk concentration automatically when the double layer interaction vanishes. On the basis of these requirements, we present a new enrichment coefficient, $\alpha$, to calculate the effective bulk ionic concentration in this work, which is defined as

$\alpha=n_{+, \mathrm{m}} / n_{\infty}$,

where $n_{+, \mathrm{m}}$ represents the counter-ion concentration at the middle of the channel. Thus, the effective bulk ionic concentration $\left(n_{\mathrm{b}}\right)$ is equal to $n_{+, \mathrm{m}}$ in value, and calculated by

$n_{\mathrm{b}}=\alpha n_{\infty}$.

The combination of Eq. 1 and Eqs. 6-9 leads to a full description of the electrochemical boundary conditions in silica nanochannels. The solution process is as follows: (i) calculate the initial boundaries $\left(\zeta_{0}\right.$ and $\left.\sigma_{0}\right)$ using Eqs. 6 and 7 through iterations by assuming $n_{\mathrm{b}}=n_{\infty}$; (ii) solve the Poisson-Boltzmann equation, Eq. 1, using the initial boundary condition ( $\zeta_{0}$ or $\sigma_{0}$ ) to obtain the counter-ion concentration at the middle of the channel $n_{+, \mathrm{m}}$, and therefore the effective bulk concentration $n_{\mathrm{b}}$; (iii) substitute $n_{\mathrm{b}}$ into Eqs. 6 and 7 and solve for the final boundary conditions ( $\zeta$ and $\sigma$ ) through another iteration process. Through such a process, the double layer interaction will influence the local ionic concentration and therefore affects the surface charge conditions.

\subsection{Electroviscous effect}

When the flow is driven by a pressure gradient, a streaming potential is established because of the ion transport. The streaming electric field is always opposite to the flow direction, and hence the net flow in the channel is diminished. This phenomenon is commonly referred as the electroviscous effect since the liquid appears to be of higher viscosity near the surfaces (Huang and Yang 2007; Li 2004; Wang et al. 2006). In this work, the streaming electric field strength is calculated by a simplified model (Li 2004)

$\mathbf{E}_{\mathrm{str}}=-\frac{\int_{0}^{h} \rho_{\mathrm{e}} \mathbf{u d} A}{A_{\mathrm{c}} \lambda_{0}+P_{\mathrm{s}} \lambda_{\mathrm{s}}}$

where $\lambda_{0}$ is the electrical conductivity of the fluid, $\lambda_{\mathrm{s}}$ is the specific surface conductivity, $A_{\mathrm{c}}$ is the effective area of the channel's cross section for the bulk conduction current, and $P_{\mathrm{S}}$ the wetting parameter of the channel (Li 2001; Ren et al. 2001). For $A_{\mathrm{c}}$, we use a piecewise function to determine its value (Ren et al. 2001; Schoch and Renaud 2005),

$A_{c}= \begin{cases}w_{\kappa}^{\frac{2}{k},} & \text { for } \frac{1}{\kappa} \leq \frac{h}{2} \\ w h, & \text { for } \frac{1}{\kappa}>\frac{h}{2}\end{cases}$

where $w$ is the channel width and $h$ the channel height. For $P_{\mathrm{s}}$, we use

$P_{\mathrm{s}}=2(w+h)$.

Although the fluid electrical conductivity $\left(\lambda_{0}\right)$ depends on the electrolyte concentration and the surface charge density (Schoch and Renaud 2005; Stein et al. 2004) and the surface conductivity $\left(\lambda_{\mathrm{s}}\right)$ may vary with the channel size (Ren et al. 2001) and the ionic transport properties in the Stern layer (Lyklema 2001), we use constant $\lambda_{0}$ and $\lambda_{\mathrm{s}}$ for simplification in this work at: $\lambda_{0}=1.42 \times$ $10^{-3} \Omega^{-1} \mathrm{~m}^{-1}$ and $\lambda_{\mathrm{s}}=1.64 \times 10^{-9} \Omega^{-1}$ ( Li and Kwok 2004; Wang et al. 2006).

\subsection{Energy conversion efficiency calculations}

In order to calculate the energy conversion efficiency of a nanofluidic device, three important transport properties have to be determined. For the pressure driven flow, the streaming conductance is defined as the streaming current per unit applied pressure (van der Heyden et al. 2005)

$c_{\mathrm{str}} \equiv I_{\mathrm{str}} / \Delta p=\frac{1}{\Delta p} \int \rho_{\mathrm{e}} u \mathrm{~d} A$,

where $I_{\text {str }}$ denotes the streaming current and $A$ the crosssection area. The hydrodynamic conductance is defined as the volume flow rate per unit applied pressure

$c_{\mathrm{h}} \equiv Q_{\mathrm{V} / \Delta p}=\frac{1}{\Delta p} \int u \mathrm{~d} A$,

where $Q_{\mathrm{V}}$ is the volume flow rate. The electroviscous effect will dent the streaming conductance and the hydrodynamic conductance more or less.

For the electroosmotic flow, the channel electrokinetic conductance is defined as the current per unit applied electrical potential difference (Stein et al. 2004) 
$c_{\mathrm{e}} \equiv I / \Delta V=\frac{1}{\Delta V}\left(\sum_{i} z_{i} e \int J_{i} \mathrm{~d} A\right)$,

where the subscript $i$ denotes the $i$ th ion species, and $J$ the ionic flux determined by the Nernst-Planck equation (Levich 1962; Lichtner 1995)

$J_{i}=-\frac{e z_{i} D_{i}}{k T} n_{i} \nabla \psi+n_{i} \mathbf{u}$,

where $z$ is the valence and $D$ the diffusivity of the ion. Another parameter, the ion mobility $\left(\mu_{\mathrm{m}}\right)$ is often used to calculate the ionic current, defined as $\mu_{\mathrm{m}, i}=e z_{i} D_{i} / k T$ (Levine et al. 1975; Stein et al. 2004). The diffusivity and mobility of ion may vary with the ionic concentration. In this study, we assume constant ion properties since it is found that the mobility variance is within $0.3 \%$ when the ionic concentration is $10^{-6}$ to $1 \mathrm{M}$ for the $\mathrm{KCl}$ solution.

Therefore, the efficiency, defined as the electrical power consumed by the load divided by the input mechanical pumping power, has a maximum value

$\eta_{\max }=\frac{\beta}{\beta+2(\sqrt{1-\beta}+1-\beta)}$,

where $\beta$ is the "figure of merit" (Morrison and Osterle 1965; Xuan and Li 2006), characterizing the coupling between electrical and fluid transport, given by

$\beta \equiv c_{\mathrm{str}}^{2} / c_{\mathrm{h}} c_{\mathrm{e}}$.

\section{Results and discussion}

After the electrochemical boundary conditions are calculated, the governing equations (1-4) are solved numerically by a lattice Poisson-Boltzmann framework (Wang et al. 2006; Wang and Chen 2007; Wang and Kang 2009) in a 2D straight nanochannel. The lattice Poisson-Boltzmann method can be regarded as a highly efficient solver for the strongly nonlinear equations governing the multiphysical electrokinetic transport (Wang et al. 2006). Different from the conventional computational methods for differential equations, the lattice evolution method employs the mesoscopic equations (for example, the Boltzmann equation) to determine macroscopic transport dynamics, and solves the governing equations by tracking the distribution functions of particle packets on lattices (Chen and Doolen 1998). The lattice Poisson-Boltzmann framework includes an electric potential evolution method on discrete lattices to solve the nonlinear Poisson-Boltzmann equation and a density evolution method on the same set of discrete lattices to solve the Navier-Stokes equations (Wang et al. 2006, 2007a, b, c). This numerical framework has been validated with analytical solutions and experimental data for micro- and nanoscale electrokinetic flows (Wang et al. 2006, 2007a, b, c; Wang and Chen 2007). In this study, we consider the flow of a $\mathrm{KCl}$ solution in a $2 \mathrm{D}$ silica nanofluidic channel. We use a $200 \times 200$ lattice system for most cases and refine the lattices when necessary to ensure that the lattice size is smaller than one-third of the Debye length $\left(\kappa^{-1}\right)$ for acceptable numerical accuracy. When the Debye length is smaller than $3 \%$ the channel width, it satisfies the "thin layer" hypothesis and the electrokinetic transport is simply calculated based on the HelmholtzSmoluchowski model (Schoch et al. 2008). The other simulation parameters, if not specified otherwise, are $\rho=$ $999.9 \mathrm{~kg} / \mathrm{m}^{3}, \varepsilon_{\mathrm{r}} \varepsilon_{0}=6.95 \times 10^{-10} \mathrm{C}^{2} / \mathrm{J} \mathrm{m}, \mu=0.889 \mathrm{mPa} \mathrm{s}$, $T=293 \mathrm{~K}, D_{\mathrm{K}^{+}}=1.96 \times 10^{-9} \mathrm{~m}^{2} / \mathrm{s}$ and $D_{\mathrm{Cl}^{-}}=2.03 \times$ $10^{-9} \mathrm{~m}^{2} / \mathrm{s}$ (Daiguji et al. 2004a, b; Wang and Chen 2007; Wang and Kang 2009).

\subsection{Validation}

In order to verify the present numerical framework, we first calculate the streaming conductance for $\mathrm{KCl}$ solution flows in a silica nanofluidic channel at $h=140 \mathrm{~nm}$, and compare the predictions with other models (constant $\zeta$ and $\sigma$ ) and the experimental data, as shown in Fig. 2. The measured streaming conductance is for a three-dimensional rectangular channel with a high aspect ratio at about 360 (van der Heyden et al. 2005). It is expected that at such a high aspect ratio, the edge effect is negligible and the 2D simulations can make a good estimation for the 3D

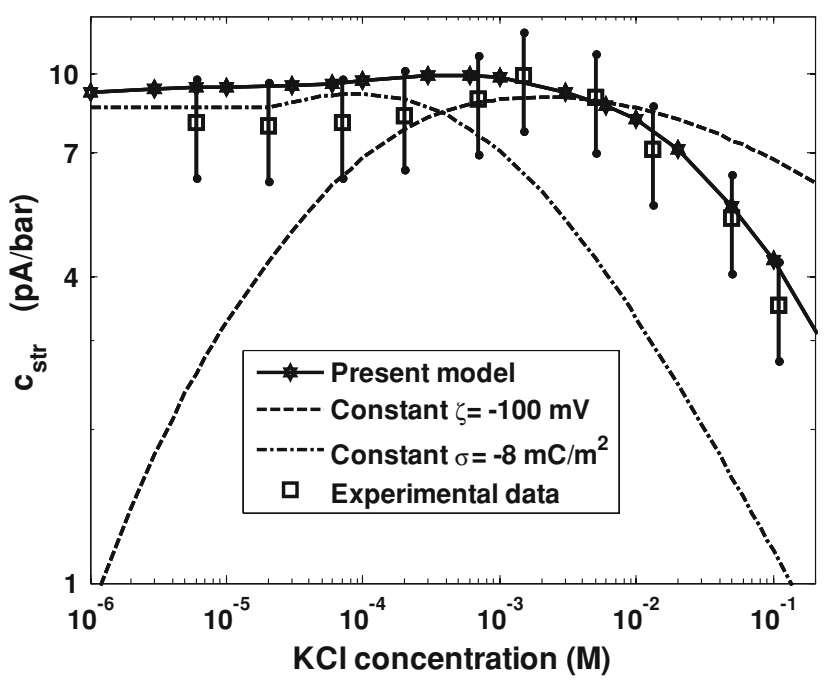

Fig. 2 Streaming conductance as a function of $\mathrm{KCl}$ concentration $\left(n_{\infty}\right)$ for a $140 \mathrm{~nm}$ high channel. The present simulation is compared with the constant zeta potential, constant charge density, and the experimental data from van der Heyden et al. (2005). The channel is $50 \mu \mathrm{m}$ wide and $4.5 \mathrm{~mm}$ long (van der Heyden et al. 2005). The chemical equilibrium parameters are $\Gamma=8 \mathrm{~nm}^{-2}, \mathrm{p} K=7.9$ and $C=2.9 \mathrm{~F} / \mathrm{m}^{2}$ from the reference Behrens and Grier (2001). The other parameters are $T=293 \mathrm{~K}$ and $\mathrm{pH}=8$ 
experiments. We use $\zeta=-100 \mathrm{mV}$ for the constant zeta potential model and $\sigma=-8 \mathrm{mC} / \mathrm{m}^{2}$ for the constant charge density model. The electroviscous effect has been considered in all the simulations. The predicted streaming conductance for a constant surface charge density $(\sigma)$ deviates from the experimental data even at low ionic concentrations because of the electroviscous effect. The modeling results using the present framework agree much better than other models with the experimental data in the entire concentration range. The streaming conductance keeps nearly constant at low salt concentrations, reaches the maximum at some moderate concentration, and decreases sharply at high ionic concentrations. It is noted that in Fig. 2 the present model still overrates the streaming conductance a little bit for low salt concentrations $\left(<10^{-4} \mathrm{M}\right)$ even though they are within the error bars. As mentioned earlier, the predictions ignored the edge effects in the third dimension. When the concentration is extremely low so that the Debye length is comparable to the channel width, the edge effects may be not negligible any more, which will weaken the streaming conductance. Another important reason is that when the salt concentration is lower than $10^{-4} \mathrm{M}$, it is virtually hard to control $\mathrm{pH}$ due to the dissolved atmospheric $\mathrm{CO}_{2}$ (Persat et al. 2009). The $\mathrm{pH}$ of the solution will actually reduce from 8 . The $\mathrm{pH}$ value effects on the transport properties of nanochannels will be discussed in the later part of this article.

\subsection{Ionic concentration effects}

The ionic concentration effect on the streaming conductance has been illustrated in Fig. 2. The streaming conductance is insensitive to the ionic concentration when the double layer interaction occurs, but will decrease sharply with the increasing salt concentration at high concentration region $\left(n_{\infty}>10^{-3} \mathrm{M}\right)$. Only the present model agrees with the experimental data for the entire concentration range. Figure 3 shows the ionic concentration effects on the electrical conductance, the hydrodynamic conductance, and therefore the energy conversion efficiency as well. Similar as the streaming conductance, both the electrical conductance and the hydrodynamic conductance are also insensitive to the ionic concentration at the low concentration range when the effective bulk salt concentration is enriched by the double layer interaction. Differently, both of them increase with the concentration at high ionic concentrations. As a result, the energy conversion efficiency decreases monotonically with the ionic concentration for a $140 \mathrm{~nm}$ high nanochannel at $\mathrm{pH}=8$, slowly at the low concentration region $\left(10^{-6}\right.$ to $\left.10^{-4} \mathrm{M}\right)$, sharply at the moderate concentration region $\left(10^{-4}-10^{-2} \mathrm{M}\right)$, and gently at the high concentration region $\left(>10^{-2} \mathrm{M}\right)$. The results indicate that a lower ionic concentration will lead to

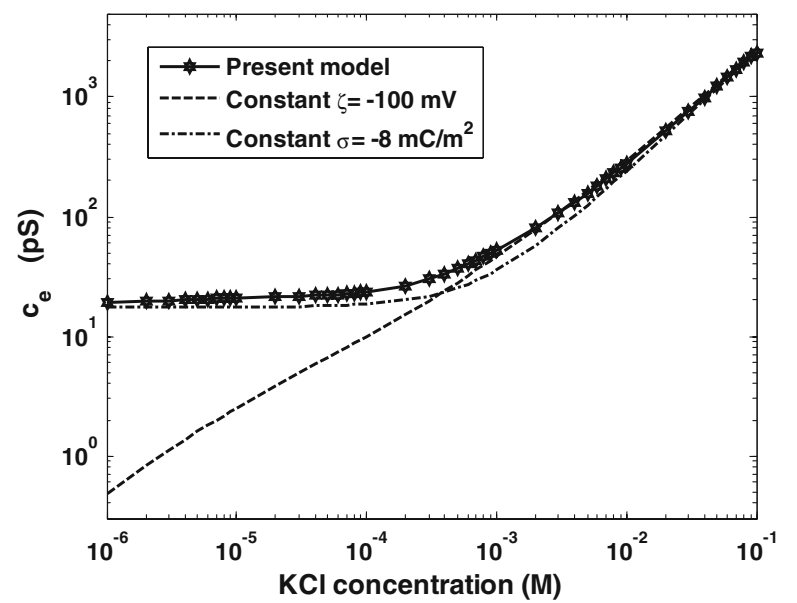

(a)

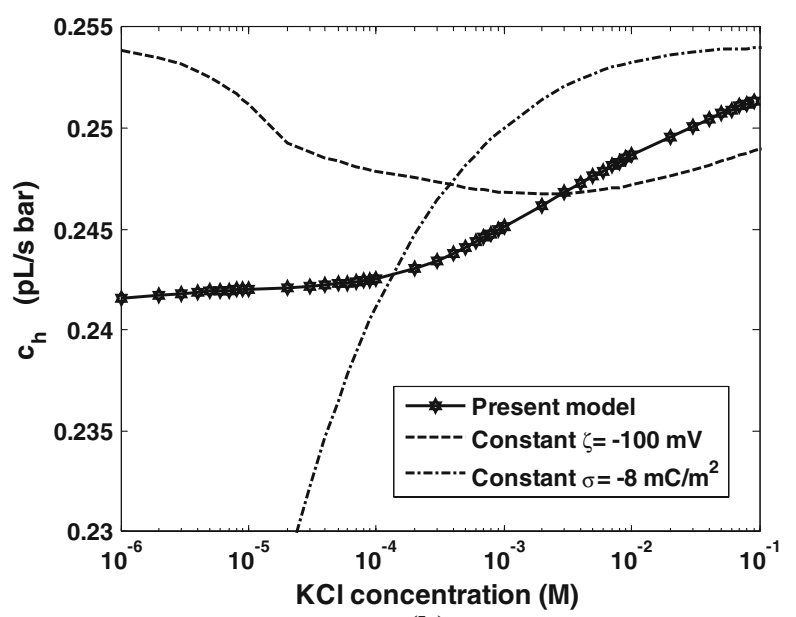

(b)

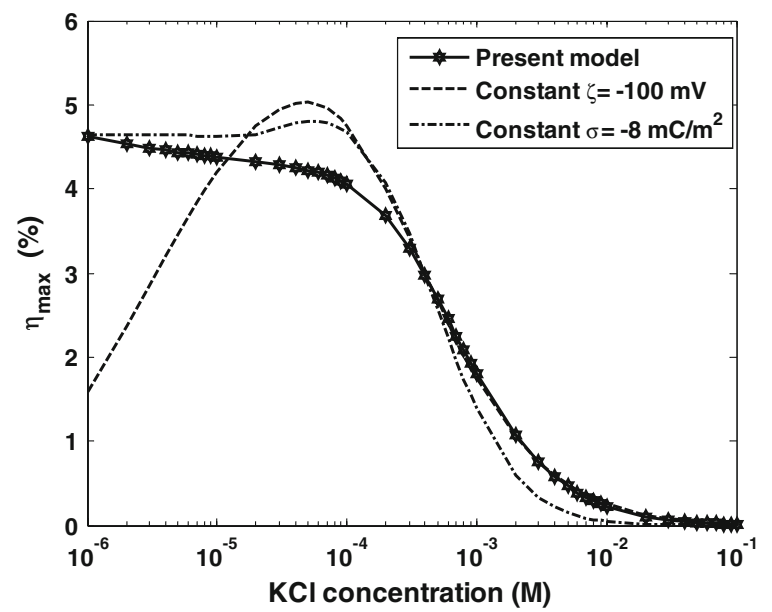

(c)

Fig. 3 Ionic concentration $\left(n_{\infty}\right)$ effects on a electric conductance, b hydrodynamic conductance, and c energy conversion efficiency. The parameters are same as those in Fig. 2

a higher energy conversion efficiency. However, as mentioned in Sect. 3.1, there are two effects, which are not involved in the modeling, to weaken the energy conversion 
at very low ionic concentrations. Therefore, in real systems, there may still be an optimal ionic concentration to maximum the energy conversion efficiency.

The modeling results based on a constant $\zeta$ and a constant $\sigma$ are also compared in Fig. 3a-c. The constant $\sigma$ assumption $\left(\sigma=-8 \mathrm{mC} / \mathrm{m}^{2}\right)$ leads to a very close prediction for the electrical conductance; however, this model overrates the hydrodynamic conductance at the high concentration range and underrates it very much at the low concentration rate. The constant $\zeta$ assumption underrates the electrical conductance when the double layers interact, but overrates the hydrodynamic conductance at the same concentration range. Both the constant $\zeta$ and $\sigma$ models predict a maximum energy conversion efficiency, respectively, at a moderate ionic concentration (around $10^{-4} \mathrm{M}$ for this case), and the constant $\zeta$ model underrates the efficiency significantly at very low ionic concentrations.

\subsection{Channel height effect}

For a given electrolyte solution and working conditions, how to design and optimize the nanofluidic devices is a very important issue. The channel height plays the key role to the energy conversion. Several researchers have studied the effects from the channel wall separation to the energy conversion efficiency using either the constant $\zeta$ (Davidson and Xuan 2008a, b; Morrison and Osterle 1965; Yang et al. 2003) or the constant $\sigma$ model (Chein et al. 2009; Danilov and Notten 2008; van der Heyden et al. 2006). Figure 4 shows the channel height effect on the energy conversion efficiency for $n_{\infty}=10^{-4} \mathrm{M}$ using the present model, compared with the other models. Each model predicts an optimal channel height to obtain the highest efficiency, which means either a too large or too narrow channel

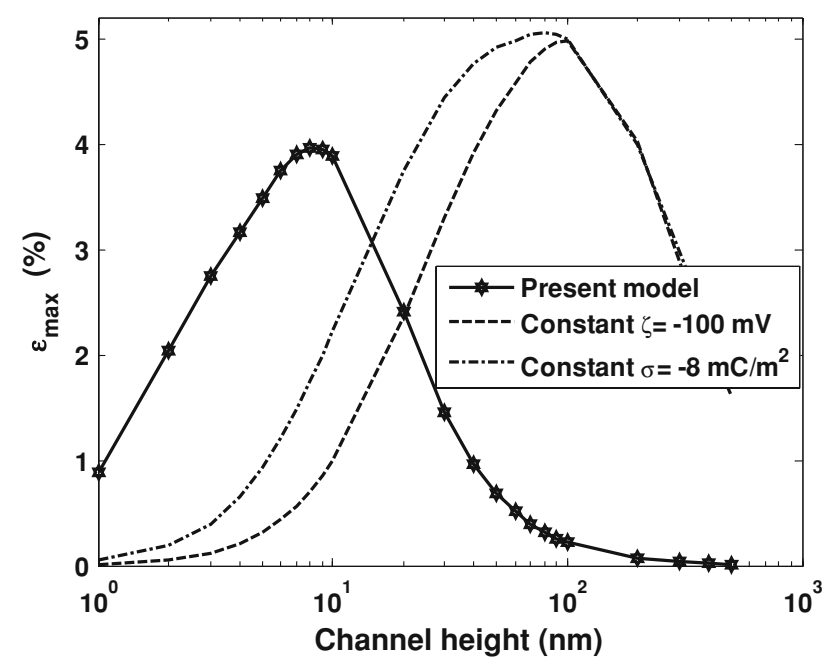

Fig. 4 Channel height effect on the energy conversion efficiency for $n_{\infty}=10^{-4} \mathrm{M}$. The other parameters are same as those in Fig. 2 would lead to a terrible decrease of the efficiency. However, the constant $\zeta$ and $\sigma$ models overate this optimal channel height by nearly one order of magnitude. For $n_{\infty}=10^{-4} \mathrm{M}$, a channel height at around $10 \mathrm{~nm}$ leads to a highest energy conversion efficiency at about $4 \%$.

\section{$3.4 \mathrm{pH}$ value effect}

As mentioned earlier, the constant $\sigma$ or $\zeta$ model is hardly able to involve the effects from the environments. Here, we investigate the $\mathrm{pH}$ and temperature effects on the energy conversion efficiency using our modified chemical equilibrium model. Figure 5 shows the streaming conductance and the energy conversion efficiency as a function of the $\mathrm{pH}$ value of $\mathrm{KCl}$ solution. Both the streaming conductance and the energy conversion efficiency decrease with the decreasing $\mathrm{pH}$ value, which proves that (1) the predictions will agree better with the experimental data in Fig. 2 at low ionic concentrations if the $\mathrm{pH}$ decrease caused by the dissolved $\mathrm{CO}_{2}$ is considered; (2) the energy conversion efficiency in Fig. 3c will drop, as a result, at some low ionic concentration, and there is an optimal ionic concentration for the highest efficiency when the other parameters are given. When the solution is acid, the efficiency is almost linearly proportional to the $\mathrm{pH}$ value. It is very interesting to find that the efficiency does not increase with the $\mathrm{pH}$ value monotonically. There is an optimal $\mathrm{pH}$ value for the maximum energy conversion efficiency. For a $\mathrm{KCl}$ concentration at $n_{\infty}=10^{-4} \mathrm{M}$ and a channel height at $h=10 \mathrm{~nm}$, the optimal $\mathrm{pH}$ value is around 8 .

\subsection{Temperature effects}

The environmental temperature effects on the zeta potential on silica surfaces and the energy conversion efficiency of nanochannels are shown in Fig. 6. The parameters of the chemical equilibrium model are the same as those in Fig. 2. We use constant properties of fluid and ions in this modeling. The results indicate that both the zeta potential and the energy conversion efficiency are in a linear relationship with the environmental temperature. The efficiency increases over $28 \%$ when the temperature varies from 273 to $333 \mathrm{~K}$. Since most of the transport properties increase, but the viscosity of aqueous solution decreases, with the temperature, the efficiency may be more enhanced if the variable properties of fluid and ions are used in this calculation.

\section{Conclusions}

A theoretical and numerical framework has been built up for modeling the multiphysiochemical transport in silica nanochannels. The surface charge boundary conditions are 


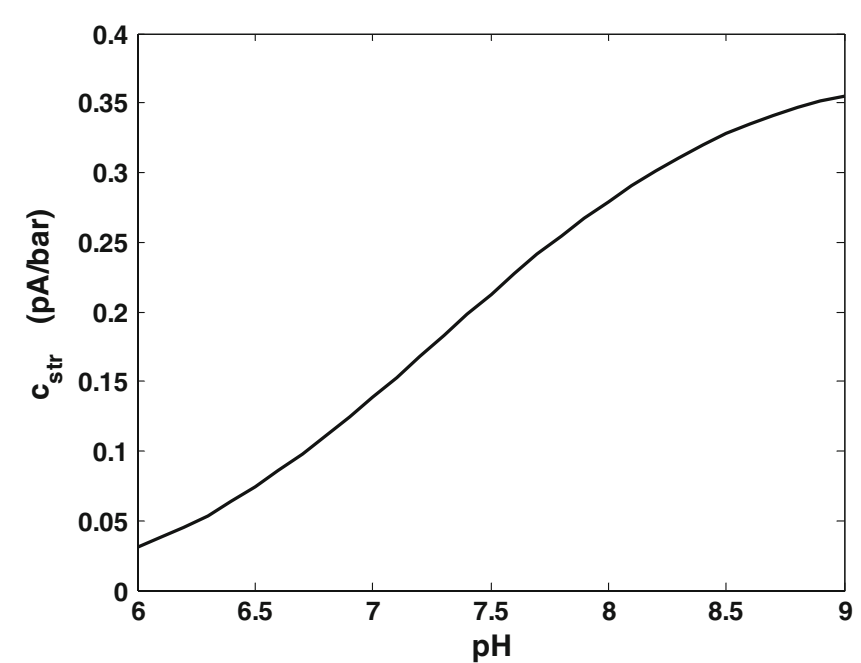

(a)

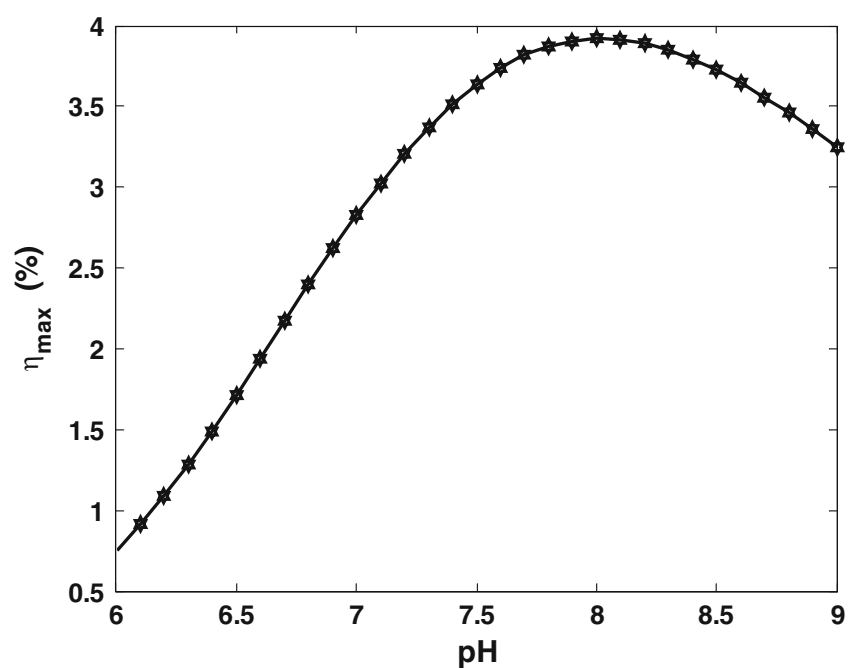

(b)

Fig. $5 \mathrm{pH}$ value effect on the streaming conductance and the energy conversion efficiency for $n_{\infty}=10^{-4} \mathrm{M}$ and $h=10 \mathrm{~nm}$. The other parameters are same as those in Fig. 2. a Streaming conductance and $\mathbf{b}$ energy conversion efficiency

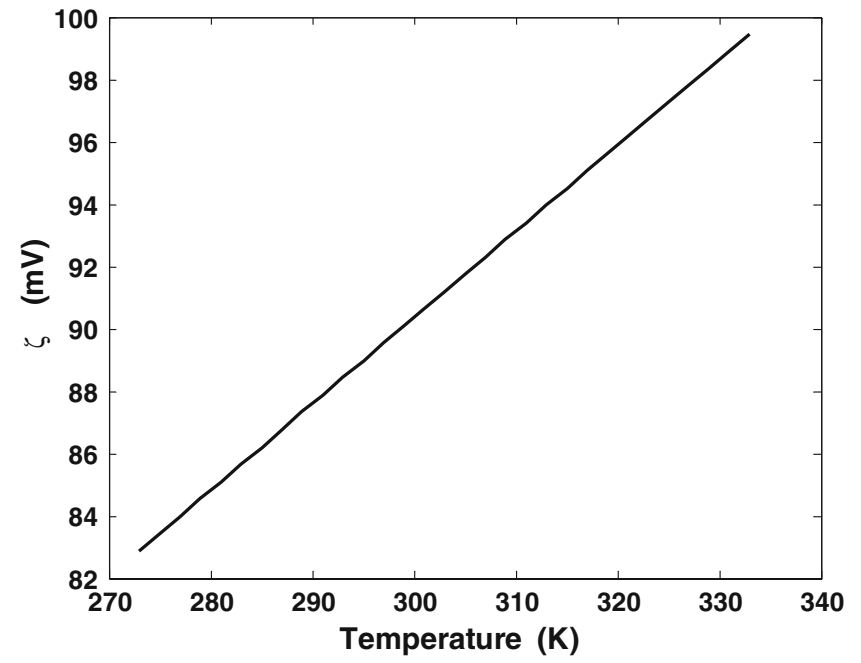

(a)

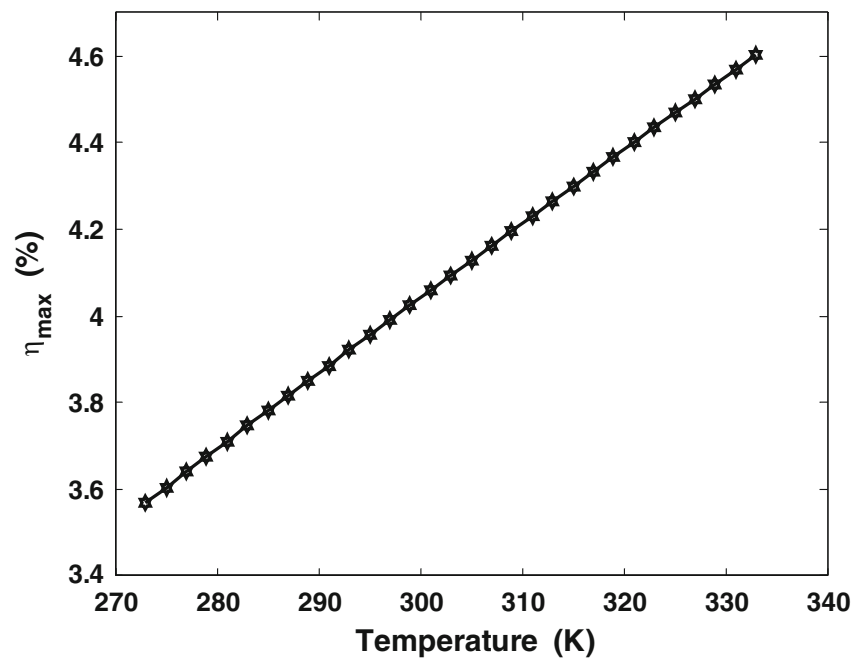

(b)

Fig. 6 Temperature effect on zeta potential on silica surfaces and the energy conversion efficiency for $n_{\infty}=10^{-4} \mathrm{M}, h=10 \mathrm{~nm}$ and $\mathrm{pH}=8$. The other parameters are same as those in Fig. 2. a Zeta potential and $\mathbf{b}$ energy conversion efficiency

calculated through the chemical equilibrium of dissociation of silanol groups, which vary with the local solid and liquid properties. The effective local bulk ionic concentration is determined by considering the EDL interactions. The electrochemomechanical energy conversion efficiency has been therefore investigated using the validated framework. The results show that the energy conversion efficiency decreases monotonically with the ionic concentration for a $140 \mathrm{~nm}$ high nanochannel at $\mathrm{pH}=8$, slowly at the low concentration region $\left(10^{-6}\right.$ to $\left.10^{-4} \mathrm{M}\right)$, sharply at the moderate concentration region $\left(10^{-4}\right.$ to $\left.10^{-2} \mathrm{M}\right)$, and gently at the high concentration region $\left(>10^{-2} \mathrm{M}\right)$. For a given ionic concentration, there is an optimal channel height to obtain the highest efficiency. The constant $\zeta$ and $\sigma$ models overate this optimal channel height by nearly one order of magnitude. For $n_{\infty}=10^{-4} \mathrm{M}$, a channel height at around $10 \mathrm{~nm}$ leads to a highest energy conversion efficiency at about $4 \%$. The efficiency does not increase with the $\mathrm{pH}$ value monotonically. There is an optimal $\mathrm{pH}$ value for the maximum efficiency when other the conditions are given. For a $\mathrm{KCl}$ concentration at $n_{\infty}=10^{-4} \mathrm{M}$ and a channel height at $h=10 \mathrm{~nm}$, the optimal $\mathrm{pH}$ value is around 8 . The energy conversion efficiency increases with the environmental temperature almost linearly for a 
constant viscosity. The present results may help to guide the design and optimization of the nanofluidic devices.

Acknowledgements This work is supported by LANL's LDRD Project 20080727PRD2, through the J. R. Oppenheimer Fellowship awarded to M. W. The authors would like to thank Prof. Stein, D. for providing the experimental data, Prof. Dekker, C., Prof. Xuan, X. C., Prof. Pennathur S and Prof. Bazant, M. for helpful discussions.

\section{References}

Abgrall P, Nguyen NT (2008) Nanofluidic devices and their applications. Anal Chem 80:2326-2341

Baldessari F (2008) Electrokinetics in nanochannels-part I. Electric double layer overlap and channel-to-well equilibrium. J Colloid Interface Sci 325:526-538

Baldessari F, Santiago JG (2009) Electrokinetics in nanochannels. Part I. Electric double layer overlap and channel-to-well equilibrium (vol 325, p 526, 2008). J Colloid Interf Sci 331:549-549

Behrens SH, Grier DG (2001) The charge of glass and silica surfaces. J Chem Phys 115:6716-6721

Butt HJ, Graf K, Kappl M (2006) Physics and chemistry of interfaces. Wiley-VCH, Weinheim

Chang CC, Yang RJ (2009) A perspective on streaming current in silica nanofluidic channels: Poisson-Boltzmann model versus Poisson-Nernst-Planck model. J Colloid Interface Sci 339:517520

Chein RY, Liao CC, Chen HJ (2009) Electrokinetic energy conversion efficiency analysis using nanoscale finite-length surfacecharged capillaries. J Power Sources 187:461-470

Chen S, Doolen GD (1998) Lattice Boltzmann method for fluid flows. Annu Rev Fluid Mech 30:329-364

Cheng LJ, Guo LJ (2007) Rectified ion transport through concentration gradient in homogeneous silica nanochannels. Nano Letters 7:3165-3171

Choi YS, Kim SJ (2009) Electrokinetic flow-induced currents in silica nanofluidic channels. J Colloid Interface Sci 333:672-678

Daiguji H, Yang PD, Majumdar A (2004a) Ion transport in nanofluidic channels. Nano Letters 4:137-142

Daiguji H, Yang PD, Szeri AJ, Majumdar A (2004b) Electrochemomechanical energy conversion in nanofluidic channels. Nano Letters 4:2315-2321

Danilov D, Notten PHL (2008) Mathematical modelling of ionic transport in the electrolyte of Li-ion batteries. Electrochim Acta 53:5569-5578

Davidson C, Xuan XC (2008a) Effects of Stern layer conductance on electrokinetic energy conversion in nanofluidic channels. Electrophoresis 29:1125-1130

Davidson C, Xuan XC (2008b) Electrokinetic energy conversion in slip nanochannels. J Power Sources 179:297-300

Duffin AM, Saykally RJ (2008) Electrokinetic Power Generation from Liquid Water Microjets. J Phys Chem C 112:17018-17022

Dufreche JF, Marry V, Malikova N, Turq P (2005) Molecular hydrodynamics for electro-osmosis in clays: from Kubo to Smoluchowski. J Mol Liq 118:145-153

Gaudin AM, Fuerstenau DW (1955) Quartz flotation with anionic collectors. Trans Am Inst Min Metall Eng 202:66-72

Huang KD, Yang RJ (2007) Electrokinetic behaviour of overlapped electric double layers in nanofluidic channels. Nanotechnology 18:115701

Joly L, Ybert C, Trizac E, Bocquet L (2004) Hydrodynamics within the electric double layer on slipping surfaces. Phys Rev Lett 93:4
Levich VG (1962) Physico-chemical hydrodynamics. Prentice-Hall, New York

Levine S, Marriott JR, Robinson K (1975) Theory of electrokinetic flow in a narrow parallel-plate channel. J Chem Soc Faraday Trans II 71:1-11

Li DQ (2001) Electro-viscous effects on pressure-driven liquid flow in microchannels. Colloids Surf 195:35-57

Li DQ (2004) Electrokinetics in microfluidics. Academic Press, Oxford

Li B, Kwok DY (2004) Electrokinetic microfluidic phenomena by a lattice Boltzmann model using a modified Poisson-Boltzmann equation with an excluded volume effect. J Chem Phys 120:947953

Lichtner PC (1995) Principles and practise of reactive transport modeling. Mater Res Soc Symp Proc 353:117-130

Lorenz CD, Crozier PS, Anderson JA, Travesset A (2008) Molecular dynamics of ionic transport and electrokinetic effects in realistic silica channels. J Phys Chem C 112:10222-10232

Lyklema J (2001) Surface conduction. J Phys Condens Matter 13:5027-5034

Morrison FA, Osterle JF (1965) Electrokinetic energy conversion in ultrafine capillaries. J Chem Phys 43:2111-2115

Pennathur S, Eijkel JCT, van den Berg A (2007) Energy conversion in microsystems: is there a role for micro/nanofluidics? Lab on a Chip 7:1234-1237

Persat A, Chambers RD, Santiago JG (2009) Basic principles of electrolyte chemistry for microfluidic electrokinetics. Part I: acid-base equilibria and $\mathrm{pH}$ buffers. Lab on a Chip 9:2437-2453

Pu QS, Yun JS, Temkin H, Liu SR (2004) Ion-enrichment and iondepletion effect of nanochannel structures. Nano Letters 4:10991103

Qiao R, Aluru NR (2004) Multiscale simulation of electroosmotic transport using embedding techniques. Int $\mathrm{J}$ Multiscale Comput Eng 2:173-188

Qiao R, Aluru NR (2005) Surface-charge-induced asymmetric electrokinetic transport in confined silicon nanochannels. Appl Phys Lett 86:143105

Ren YQ, Stein D (2008) Slip-enhanced electrokinetic energy conversion in nanofluidic channels. Nanotechnology 19:195707

Ren LQ, Li DQ, Qu WL (2001) Electro-viscous effects on liquid flow in microchannels. J Colloid Interface Sci 233:12-22

Schoch RB, Renaud P (2005) Ion transport through nanoslits dominated by the effective surface charge. Appl Phys Lett $86: 253111$

Schoch RB, Han JY, Renaud P (2008) Transport phenomena in nanofluidics. Rev Mod Phys 80:839-883

Squires TM, Quake SR (2005) Microfluidics: fluid physics at the nanoliter scale. Rev Mod Phys 77:977-1026

Stein D, Kruithof M, Dekker C (2004) Surface-charge-governed ion transport in nanofluidic channels. Phys Rev Lett 93:035901

van der Heyden FHJ, Stein D, Dekker C (2005) Streaming currents in a single nanofluidic channel. Phys Rev Lett 95:116104

van der Heyden FHJ, Bonthuis DJ, Stein D, Meyer C, Dekker C (2006) Electrokinetic energy conversion efficiency in nanofluidic channels. Nano Letters 6:2232-2237

van der Heyden FHJ, Bonthuis DJ, Stein D, Meyer C, Dekker C (2007) Power generation by pressure-driven transport of ions in nanofluidic channels. Nano Letters 7:1022-1025

Wang M, Chen S (2007) Electroosmosis in homogeneously charged micro- and nanoscale random porous media. J Colloid Interface Sci 314:264-273

Wang M, Chen SY (2008) On applicability of Poisson-Boltzmann equation for micro- and nanoscale electroosmotic flows. Commun Comput Phys 3:1087-1099

Wang M, Kang Q (2009) Electrokinetic transport in microchannels with random roughness. Anal Chem 81:2953-2961 
Wang JK, Wang M, Li ZX (2006) Lattice Poisson-Boltzmann simulations of electro-osmotic flows in microchannels. J Colloid Interface Sci 296:729-736

Wang M, Liu J, Chen S (2007a) Electric potential distribution in nanoscale electroosmosis: from molecules to continuum. Mol Simul 33:1273-1277

Wang M, Liu J, Chen S (2007b) Similarity of electroosmotic flows in nanochannels. Mol Simul 33:239-244

Wang MR, Wang JK, Chen SY (2007c) Roughness and cavitations effects on electro-osmotic flows in rough microchannels using the lattice Poisson-Boltzmann methods. J Comput Phys 226: 836-851
Westall J, Hohl H (1980) Comparison of electrostatic models for the exide-solution interface. Adv Colloid Interface Sci 12:265-294

Xie YB, Wang XW, Xue JM, Jin K, Chen L, Wang YG (2008) Electric energy generation in single track-etched nanopores. Appl Phys Lett 93:163116

Xuan XC, Li DQ (2006) Thermodynamic analysis of electrokinetic energy conversion. J Power Sources 156:677-684

Yang J, Lu FZ, Kostiuk LW, Kwok DY (2003) Electrokinetic microchannel battery by means of electrokinetic and microfluidic phenomena. J Micromech Microeng 13:963-970 